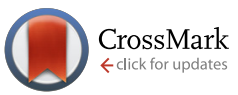

Cite this: Med. Chem. Commun., 2016, 7, 1546

Received 21st March 2016, Accepted 4th May 2016

DOI: $10.1039 / \mathrm{c} 6 \mathrm{md} 00163 \mathrm{~g}$

www.rsc.org/medchemcomm

\title{
Synthetic analogues of marine alkaloid clathrodin differently induce phosphatidylserine exposure in monocytic cancer cells then in cancer stem cell linestt
}

\author{
Dominik Nabergoj, $\S^{\text {ab }}$ Sanja Vrbek, $\S^{\text {ab }}$ Nace Zidar, ${ }^{a}$ Tihomir Tomašić, ${ }^{a}$ Danijel Kikelj, ${ }^{a}$ \\ Lucija Peterlin Mašič ${ }^{a}$ and Christian D. Muller ${ }^{\star b}$
}

\begin{abstract}
Activation of apoptosis in cancer cells could stop the development of several cancers. Through testing in different human cancer cell lines (blood, liver, pancreas) and cancer stem cell lines (pancreas and testis), a cytomic approach was performed to investigate kinetic differences in the mechanism of proapoptotic activity induced by four synthetic analogues of clathrodin - an alkaloid isolated from Caribbean sea sponge. Active on the tested cancer stem cell lines, analogue 5 interestingly shows a differential response in apoptosis induction in the presence of pan caspase inhibitor Z-VAD-fmk in monocytic THP-1 cells. Thus in monocytic cells, contrary to cancer stem cells, this caspase independent mechanism could reflect either a scramblase modulation, a disturbed bidirectional trafficking of the membrane between the surface and cy-

toplasm or a necroptosis induction.
\end{abstract}

\section{Introduction}

Elucidating the mechanism of action of molecules inducing apoptosis is an important step to understand the mechanisms of resistance to any pro-apoptotic signal. Such resistance facilitates the survival of pro-inflammatory cells favoring the perpetuation of chronic inflammation and furthermore the development of associated cancers. ${ }^{1}$ This work is focused on understanding the activation of apoptosis in cancer. Pharmacological tools, derived from marine alkaloid clathrodin (MAREX project "Exploring Marine Resources for Bioactive Compounds: From Discovery to Sustainable Production and Industrial Applications 2010-2014" (www.marex. fi) and COST Action CM1106) enabled us to study in detail an original model of apoptosis induced anticancer activity. Our results suggest that the induced phosphatidylserine (PS) externalization reflects trafficking of lysosomes to the cell surface ${ }^{2}$ rather than a simple enzymological process. ${ }^{3}$

\footnotetext{
${ }^{a}$ Faculty of Pharmacy, University of Ljubljana, Aškerčeva 7, 1000 Ljubljana, Slovenia

${ }^{b}$ Laboratoire d'Innovation Thérapeutique, UMR 7200, Faculté de Pharmacie, Université de Strasbourg, Illkirch, France.E-mail: cdmuller@unistra.fr; Tel: +33688275739

$\dagger$ The authors declare no competing interests.

\$ Electronic supplementary information (ESI) available. See DOI: 10.1039/ c6md00163g

$\S$ These authors contributed equally to this work.
}

Like some natural products, marine bioactive compounds generally don't possess favorable physicochemical properties and their structure has to be modified in order to obtain analogues with improved potency, selectivity and favorable pharmacokinetic properties. ${ }^{4}$ Clathrodin (1a) is a pyrrole-2aminoimidazole alkaloid (Fig. 1) isolated from the Caribbean sea sponge Agelas clathrodes $^{5}$ and is structurally related to its brominated analogues oroidin (1b) from the sponge Agelas oroides $^{6,7}$ and hymenidin (1c) from the sponge Hymeniacidon perlevis. ${ }^{8}$ A broad spectrum of reported bioactivities for marine alkaloids clathrodin, oroidin and hymenidin include voltage-gated sodium channel blocking, ${ }^{9,10}$ antimicrobial, ${ }^{11}$ antihistaminic, ${ }^{12}$ antiserotoninergic, ${ }^{8}$ antimuscarinic, ${ }^{13}$ and cytotoxic ${ }^{5}$ properties. Such a broad spectrum of biological activities stimulated our interest in the design and synthesis of libraries of analogues of marine alkaloids $1 \mathrm{a}-\mathbf{c}^{\mathbf{1 4 , 1 5}}$ and to monitor their proapoptotic activities in human cancer cells of different origins.

In a previous study, ${ }^{15}$ clathrodin, oroidin, hymenidin and a library of 96 synthetic analogues from three structural families were screened for their apoptosis inducing activities against the human hepatocellular carcinoma HepG2 cell line. Although the tested marine alkaloids were found to be only weakly active, their most potent indole-based analogues 2-5, obtained by rigidization in the central part of the parent alkaloids and annelation of a phenyl ring to the pyrrole moiety, ${ }^{16,17}$ exhibited $\mathrm{EC}_{50}$ values of $13-20 \mu \mathrm{M}$ for HepG2 cell line apoptosis induction and $\mathrm{EC}_{50}$ values of $20-24 \mu \mathrm{M}$ for 


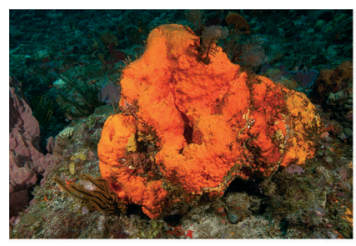

Agelas clathrodes

doris.ffessm.fr/ref/specie/1155

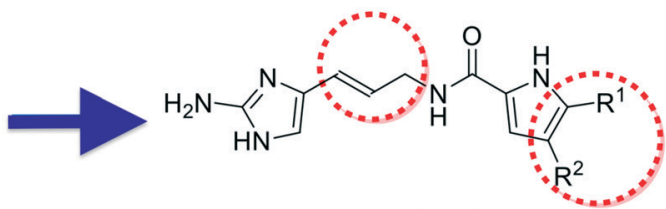

1a clathrodin $\left(\mathrm{R}^{1}=\mathrm{R}^{2}=\mathrm{H}\right)$

1b hymenidin $\left(\mathrm{R}^{1}=\mathrm{H}, \mathrm{R}^{2}=\mathrm{Br}\right)$

$1 c$ oroidin $\left(R^{1}=R^{2}=B r\right)$ med-chem

modification

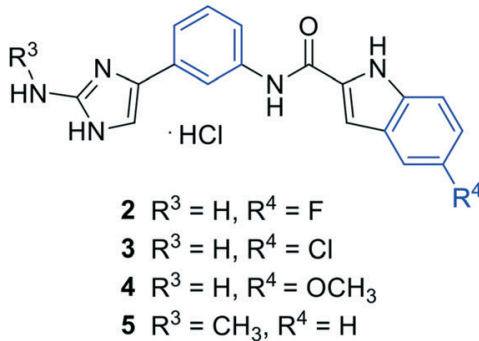

Fig. 1 Clathrodin and synthetic analogs. Clathrodin (1a) is a pyrrole-2-aminoimidazole alkaloid from the Caribbean sea sponge Agelas clathrodes structurally related to its brominated analogues hymenidin (1b) from the sponge Hymeniacidon perlevis and oroidin (1c) from the sponge Agelas oroides. Indole-based analogues $\mathbf{2 - 5}$ are obtained by rigidization in the central part of the parent alkaloids and annelation of a phenyl ring to the pyrrole moiety.

apoptosis induction in the acute monocytic leukemia THP-1 cell line. Through cell cycle analysis, we found that compounds $2-5$ induce apoptosis in THP- 1 cells at $25 \mu \mathrm{M}$ concentration. ${ }^{15}$ These results stimulated us to perform a more comprehensive analysis of the mechanism of proapoptotic activity of compounds $2-5$ in different cancer phenotypes (pancreas, liver and blood) and cancer stem cell lines (testis and pancreas), an investigation presented herein. Time course experiments revealed a different way of inducing phosphatidylserine (PS) translocation onto the cell surface. Thus, by activation of an enzyme part of the flippase family e.g. the scramblase, ${ }^{3}$ compounds $2-5$ could disturb bidirectional trafficking of membrane components between the cell surface and cytoplasm following a model where PS externalization reflects $\mathrm{Ca}^{2+}$-mediated trafficking of many vesicles to the cell surface. ${ }^{2}$

\section{Materials and methods}

\section{Compounds 2-5}

The clathrodin analogs $2,{ }^{16} 3,{ }^{16} 4$ (ref. 16) and 5 (ref. 17) were prepared as described by us previously. The purity of the tested compounds was $\geq 95 \%$.

\section{Cell lines and culture conditions}

NT2/D1 (CRL-1973), AsPC-1 (CRL-1682) and BJ (CRL-2522) cell lines, all purchased from ATCC (LGC Standards, Molsheim, France), were cultivated in physiological nutrientrich DMEM-based media (Sigma-Aldrich, Saint-QuentinFallavier, France), supplemented with $10 \%$ (v/v) fetal bovine serum (BioWhittaker, Verviers, Belgium), $2 \mathrm{mM}$ glutamine, $50 \mu \mathrm{M}$ non-essential amino acids, $50 \mathrm{U} \mathrm{ml}^{-1}$ penicillin and 50 $\mu \mathrm{g} \mathrm{ml}{ }^{-1}$ streptomycin (Sigma-Aldrich). THP-1 (TIB-202) and BxPC-3 (CRL-1687) cells were grown in RPMI 1640 medium with $2 \mathrm{mM}$ L-alanyl-L-glutamine additionally supplemented with $10 \%(\mathrm{v} / \mathrm{v})$ fetal bovine serum, $50 \mathrm{U} \mathrm{ml}^{-1}$ penicillin and $50 \mu \mathrm{g} \mathrm{ml}^{-1}$ streptomycin (Sigma-Aldrich). HepG2 (HB-8065) cell line was maintained in MEM media supplemented with $10 \%(\mathrm{v} / \mathrm{v})$ fetal bovine serum, $50 \mathrm{U} \mathrm{ml}^{-1}$ penicillin, $50 \mu \mathrm{g} \mathrm{ml}{ }^{-1}$ streptomycin (Sigma-Aldrich) and $2 \mathrm{mM}$ glutamine. Prior to treatment, cells were grown up to $80 \%$ confluency at $37{ }^{\circ} \mathrm{C}$ under humidified atmosphere $\left(5 \% \mathrm{CO}_{2}\right)$.

\section{Cell treatment}

Compounds 2-5 were diluted at $10 \mathrm{mM}$ in DMSO and cells were treated with appropriate working concentrations premixed into cell culture medium. The highest concentration of DMSO (in treated and control cells) never exceeded $0.25 \%(\mathrm{v} / \mathrm{v})$ to avoid any side effects, like toxicity or differentiation induction. Experiments with the pan-caspase inhibitor Z-VAD-FMK (obtained from BD Biosciences Pharmingen, San Diego, CA, USA) were carried out according to the manufacturer's instructions.

\section{Apoptosis rate analysis by annexin $\mathrm{V}$ binding assay}

Revealing the externalization of phosphatidylserine and labeling the dead cell nucleus were used to assess apoptosis after cells were cultured and treated or not with compounds 2-5. Except for the non-adherent THP-1 cells, all other cell lines were washed with PBS, trypsinized, centrifuged and resuspended in the first wash supernatant to preserve all nonadherent apoptotic cells. A minimum of 2000 cells were acquired and analyzed on InCyte (Guava/Millipore/Merck, CA, USA). Apoptosis rates were assessed by capillary cytometry (Guava EasyCyte Plus, Millipore Merck) after Annexin V-FITC (ImmunoTools, Germany) and PI (MiltenyiBiotec Inc., USA) labeling according to the manufacturer's recommendations. Areas were drawn around single cell populations on forward scatter (FSC) versus side scatter (SSC) dot plots so as to exclude any debris. Cytometer performance was checked weekly using the Guava easyCheck Kit 4500-0025 (Merck/Millipore/ Guava Hayward, CA, USA).

\section{Cell cycle phase distribution analysis and quantification of hypo diploid sub-G1 cell population}

Cells were cultured at a density of $10^{5}-10^{6}$ cells per ml depending on the cell line, in accordance with ATCC's recommendations. After $24 \mathrm{~h}$, cells were incubated with compounds $2-5(25 \mu \mathrm{M})$ for different time periods in $6,12,24$ or 96 well plates depending on the cell line phenotype. Then 
cells were washed with PBS, re-suspended in 70\% ethanol and placed for $24 \mathrm{~h}$ at $-20{ }^{\circ} \mathrm{C}$. After centrifugation $(5 \mathrm{~min}$, $400 g$ ), cell pellets were washed twice with PBS then resuspended in $500 \mu \mathrm{l}$ of PBS, incubated in FxCycle PI/RNase Staining Solution (Life technologies, Thermo Fisher Scientific Inc. USA) and kept in the dark at room temperature for 30 min. Cellular DNA content was then assessed by capillary cytometry on a Guava EasyCyte Plus HP cytometer (EMD Millipore Corporation, MA, USA). A minimum of 10000 cells per sample was acquired and analyzed on the InCyte software. The percentage of cells in $\mathrm{G}_{0} / \mathrm{G}_{1}, \mathrm{~S}, \mathrm{G}_{2} / \mathrm{M}$ and sub-G1 was determined from DNA content histograms.

\section{Caspase-3/7 and caspases 8 and 9 activity determinations}

Cells were cultivated as previously described. Apoptosis wash buffer and caspase reagent working solution were prepared according to the manufacturer's instructions (Merck Millipore, Darmstadt, Germany). After 24 hours, cells were incubated for different times with or without compounds 2-5 (25 $\mu \mathrm{M})$. After trypsinization, except for non-adherent THP-1 cells, cells were collected by centrifugation and handled according to the manufacturer's instructions (Merck Millipore, Caspase 3/7 FAM, Caspase 9 SR, Caspase 8 FAM, Guava
Kits for Flow Cytometry). A minimum of 5000 cells was acquired on the capillary cytometer then analyzed on InCyte.

\section{Results}

\section{Apoptosis induction activities of clathrodin analogues 2-5}

Previous results of proapoptotic activity in cancer cells of several analogues of clathrodin ${ }^{15}$ lead us to test four best performing compounds in additional human cancer and cancer stem cell (CSC) cell lines. Table 1 allows the comparison of the $\mathrm{EC}_{50}$ values of the four analogues for apoptosis induction in different cell phenotypes. When considering CSCs of testis (NT2/D1) or pancreatic origin (AsPC-1), micromolar $\mathrm{EC}_{50}$ values were observed showing anticancer potency. For other cancerous cell lines of pancreatic (BxPC-3), hepatic (HepG2) or hematopoietic (THP-1) phenotype, similar potencies were obtained to the non-cancerous human fibroblasts cell line (BJ). All four analogs showed similar effectiveness $\left(\mathrm{EC}_{50}\right.$ values in the range of $\left.11-16 \mu \mathrm{M}\right)$ in NT2/D1 CSCs. Among all the tested cell phenotypes, the monocytic THP-1 cell line was the one showing particularities in kinetic responsiveness, making our compounds original pharmacological tools as further explained in the next paragraph. Possessing a 2-( $N$-methylamino) imidazole moiety with no

Table 1 Clathrodin analogues apoptosis induction activity ${ }^{a}$

\begin{tabular}{|c|c|c|c|c|c|c|c|}
\hline \# & Structure & NT2/D1 & AsPC-1 & BxPC-3 & HepG2 & $\mathrm{BJ}$ & THP-1 \\
\hline (2) & & $13 \pm 5$ & $27 \pm 3$ & $28 \pm 4$ & $13 \pm 7$ & $16 \pm 6$ & $23 \pm 4$ \\
\hline (3) & & $16 \pm 8$ & $19 \pm 1$ & $25 \pm 3$ & $16 \pm 6$ & $20 \pm 3$ & $24 \pm 5$ \\
\hline (4) & & $16 \pm 1$ & $52 \pm 3$ & $53 \pm 12$ & $18 \pm 1$ & $31 \pm 17$ & $20 \pm 2$ \\
\hline (5) & & $11 \pm 2$ & $32 \pm 9$ & $58 \pm 14$ & $20 \pm 5$ & $21 \pm 9$ & $24 \pm 1$ \\
\hline
\end{tabular}

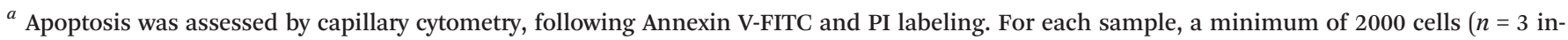

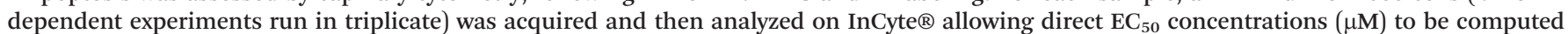

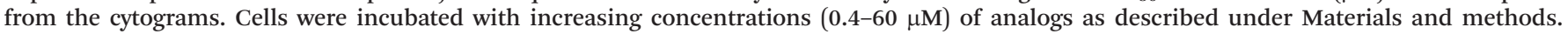

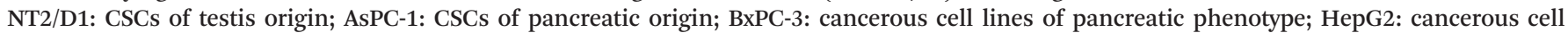

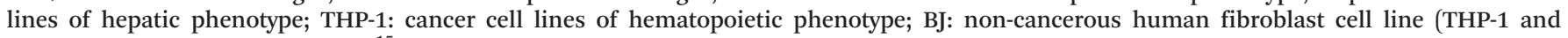
HEPG-2 data published previously ${ }^{15}$ ).
} 
NT2/D1

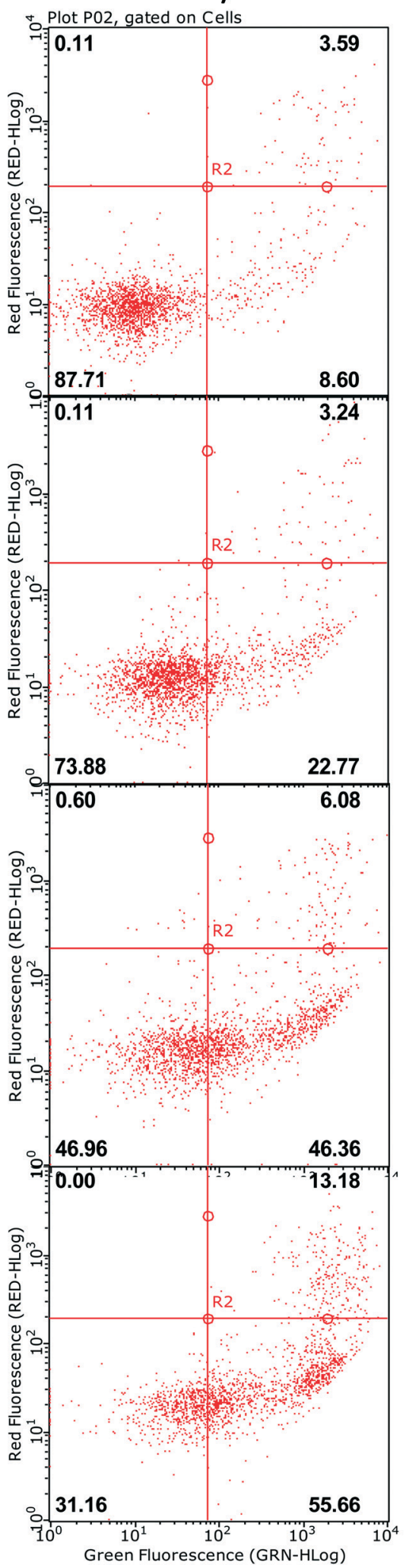

THP-1

Compound \# 5

$[25 \mu \mathrm{M}]$

$1 \mathrm{H}$

$3 \mathrm{H}$

$5 \mathrm{H}$

$7 \mathrm{H}$

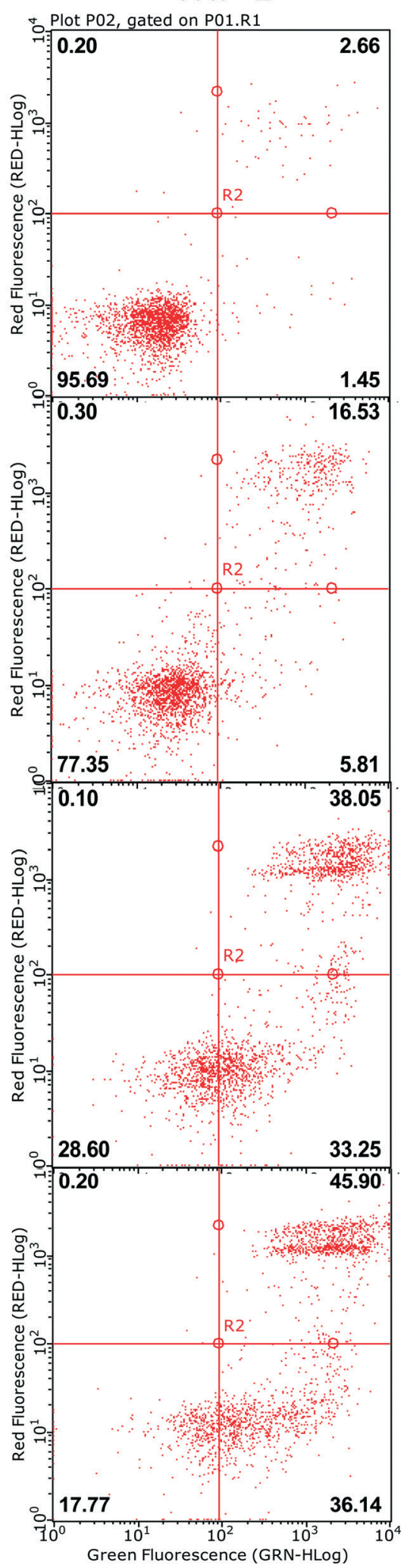

Fig. 2 Time course response for proapoptotic activity of compound 5 on NT2/D1 and THP-1. These representative dot plots were obtained after time-dependent treatment with compound $5[25 \mu \mathrm{M}]$ of NT2/D1 and THP-1 cells. Cells were labeled with Annexin V (green fluorescence (GRNHLog))/PI (red fluorescence (RED-HLog)) as described under Materials and methods. The numbers indicated in each quadrant represent the different apoptotic states expressed as \% of the total cell number (2000 events per sample). 
substitution in the indole ring and displaying a suitable proapoptotic activity on NT2/D1 cancer stem cells $\left(\mathrm{ED}_{50}=11\right.$ $\pm 2 \mu \mathrm{M}$ ), compound 5 was chosen for further mechanistic experiments on NT2/D1 CSC and THP-1 cell lines.

\section{Differential activity of compound 5 on monocytic cancer phenotype}

Time course experiments (Fig. 2) indicate that after 3 hours, compound 5 was able to induce $17 \%$ of THP-1 cells to die by apoptosis when only $3 \%$ of NT2/D1 were affected (cells in the upper right quadrant). If after 5 hours this percent was doubled, after 7 hours, 4 times more THP-1 cells were dead. This kinetic difference suggests a divergence in targets involved in cell death for THP-1 cells, considering all other tested cell types were responding as the NT2/D1 phenotype (results for the other cell types not shown). With THP-1 cells being of immune phenotype, necroptosis is a possible mechanism to be involved here, as it is characterized as a component of inflammatory diseases when im- mune cells undergo "cellular suicide". ${ }^{18,19}$ Thus, further experiments done in the presence of a pan-caspase inhibitor should help us to understand these kinetic dissimilarities in PS externalization. ${ }^{19}$

\section{Compound 5 induces sub-G1 population in both THP-1 and NT2/D1 cells}

If an increased number of hypodiploid sub-G1 cells can be observed when teratocarcinomal cancer stem-like cells NT2/ D1 and monocytic cells THP-1 are exposed to compound 5, death by apoptosis cannot be excluded as the cellular DNA content is reduced even if kinetic response profiles are different to extracellular PS exposure previously demonstrated in Fig. 2 by accumulation of cells in the upper right quarter of the cytograms (PS + PI positives cells). Induction of DNA fragmentation was observed in both cell lines (Fig. 3) with, this time, no kinetic distinctions. Even a delay for THP-1 DNA fragmentation was noticeable when compared to the fast response rate for PS exposure (see Fig. 2).

\section{A NT2/D1}
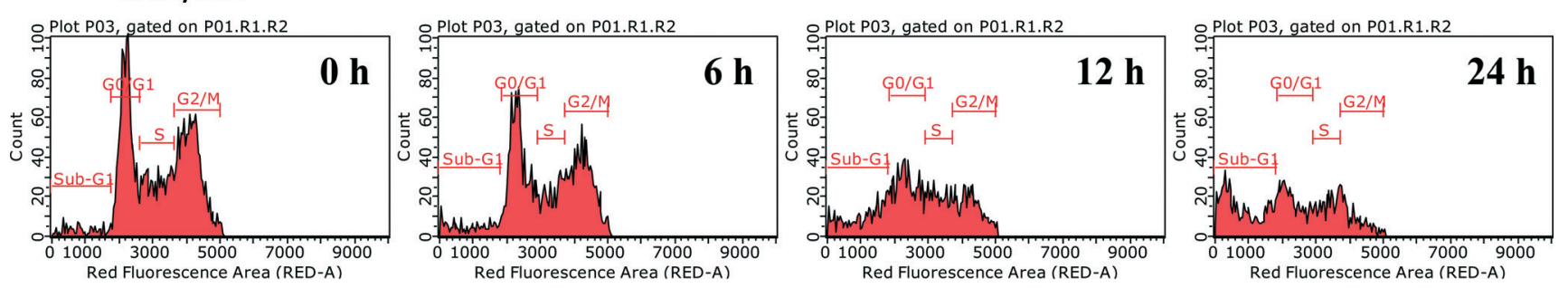

\section{B THP-1}
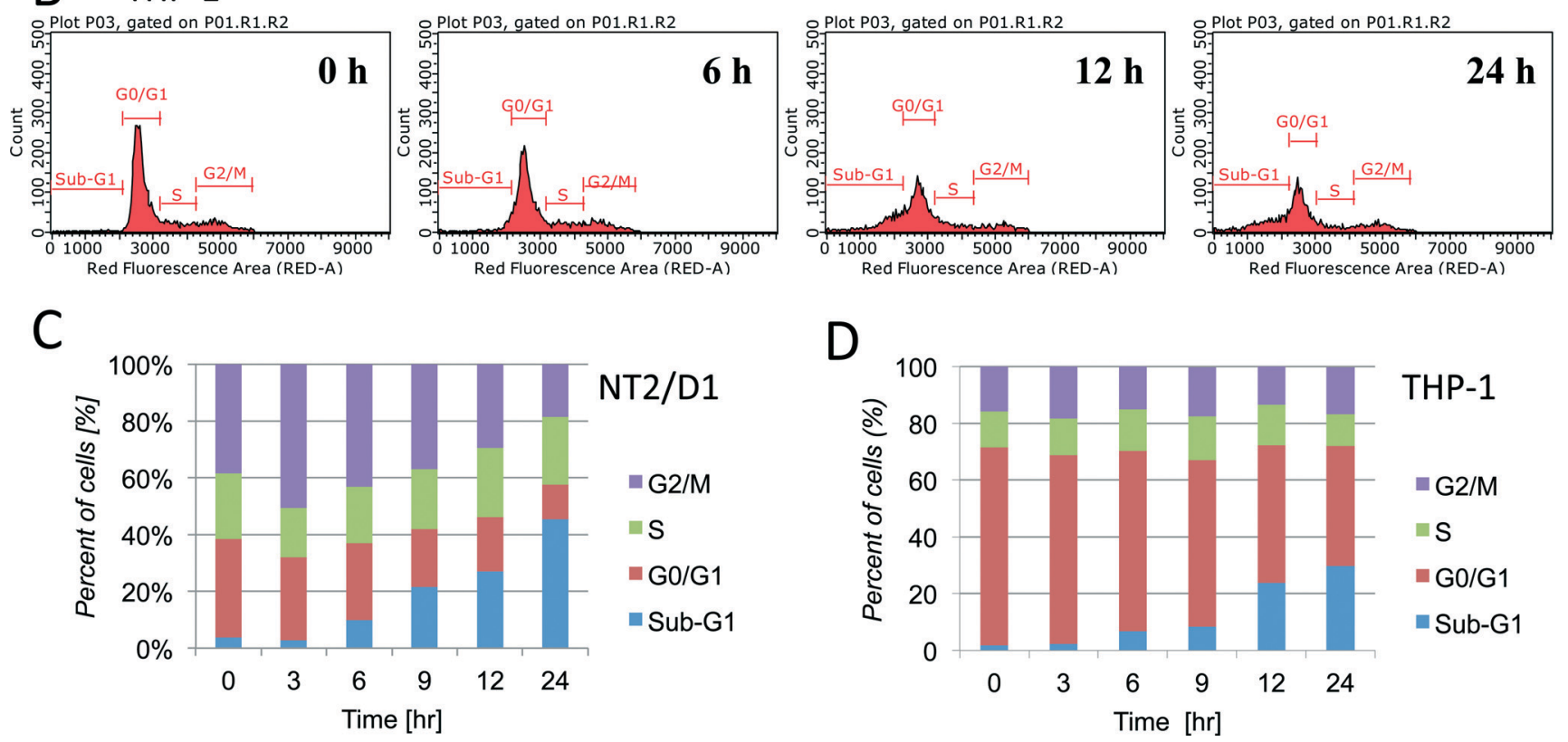

Fig. 3 Cell cycle analyses for proapoptotic activity of compound 5 on NT2/D1 and THP-1. Cell cycle distribution was assessed by capillary cytometry. This figure shows representative time course of DNA content histograms (10000 events per sample) for (A) NT2/D1 and (B) THP-1 cell in the presence of $25 \mu \mathrm{M}$ of compound 5. (C) and (D) recapitulate for each cell line, in a bar graph format, the distribution in sub-G1, G0/G1, S or G2/M phases; the number of cells in each mitosis phase was computed and expressed as \% of the total cellular population. 
Compound 5 induces apoptosis in cancer stem-like cells and monocytic cells via a caspase-dependent mechanism

According to the biochemical classification of cell death, apoptosis can be divided, due to different mechanistic pathways, into extrinsic and intrinsic apoptosis. ${ }^{20}$ Extrinsic apoptosis is potentially coupled with intrinsic apoptosis since caspase 8 activation can also cleave and activate pro-apoptotic protein Bid and cause permeabilization of the mitochondrial outer membrane, which in turn activates caspase 9 and later on also caspases $3 / 7 .^{21}$ Activation of caspases $3 / 7$, one of the most significant apoptotic effector caspases, leads to final execution of apoptotic cell death since activated caspase 3 activates caspase-activated DNase (CAD) which causes fragmentation of DNA and chromatin condensation. Furthermore, caspase 3 is also responsible for reorganization of cytoskeleton and formation of apoptotic bodies. Data in Fig. 4 shows activation of caspases $3 / 7$ in NT2/D1 and THP-1 cell lines, starting already after 3 hours of incubation with compound
5, as expected by detection of DNA fragmentation. Caspase 8 activation was observed prior to activation of caspase 9, indicating that the extrinsic pathway is activated in both cell lines (Fig. 4). Again, no kinetic differences were observed here between the two cell lines. We further examined the effects of a caspase inhibitor on proapoptotic activity in teratocarcinoma cells.

Compound 5 induces PS exposure in monocytic cells via a caspase-independent mechanism

As shown in Fig. 5, the pan-caspase inhibitor Z-VAD-FMK was able to fully countervail the exposure of PS in NT2/D1 cancer stem cells: after 4 hours, only a few cells migrated to the quarters of the cytograms, where PS + PI positive cells accumulate. On the contrary, annexin V was able to stain $82 \%$ of THP-1 cells after the same time period as all the cells remained in the right part of the cytogram. Knowing that necroptosis is a well-defined viral defense mechanism which

\section{NT2/D1}

\section{Caspase $3 / 7$}

\section{THP-1}
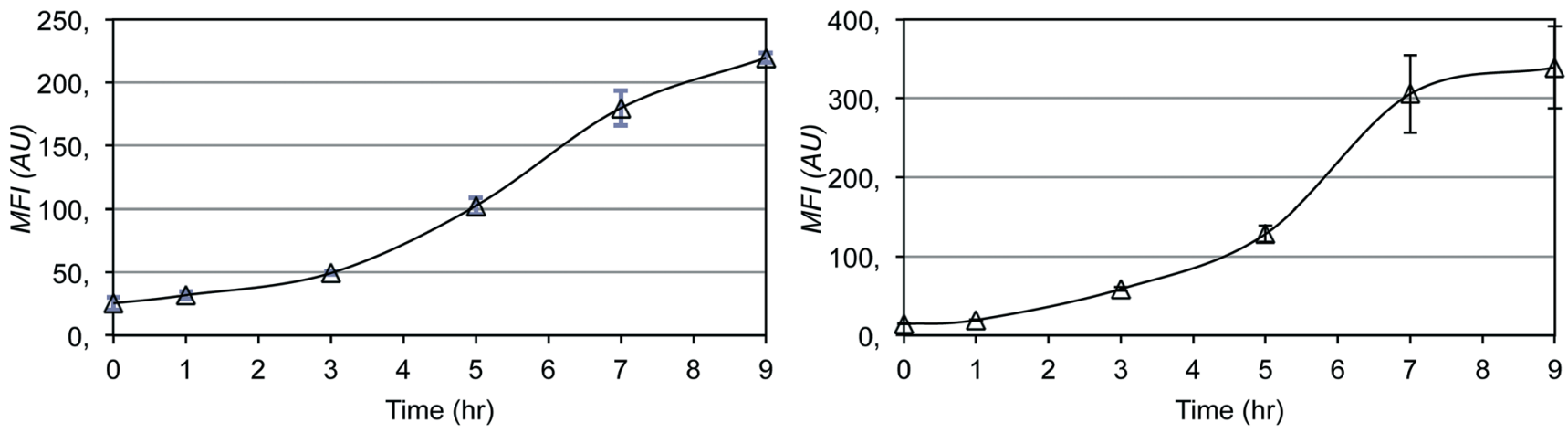

\section{Caspase 8 and 9}
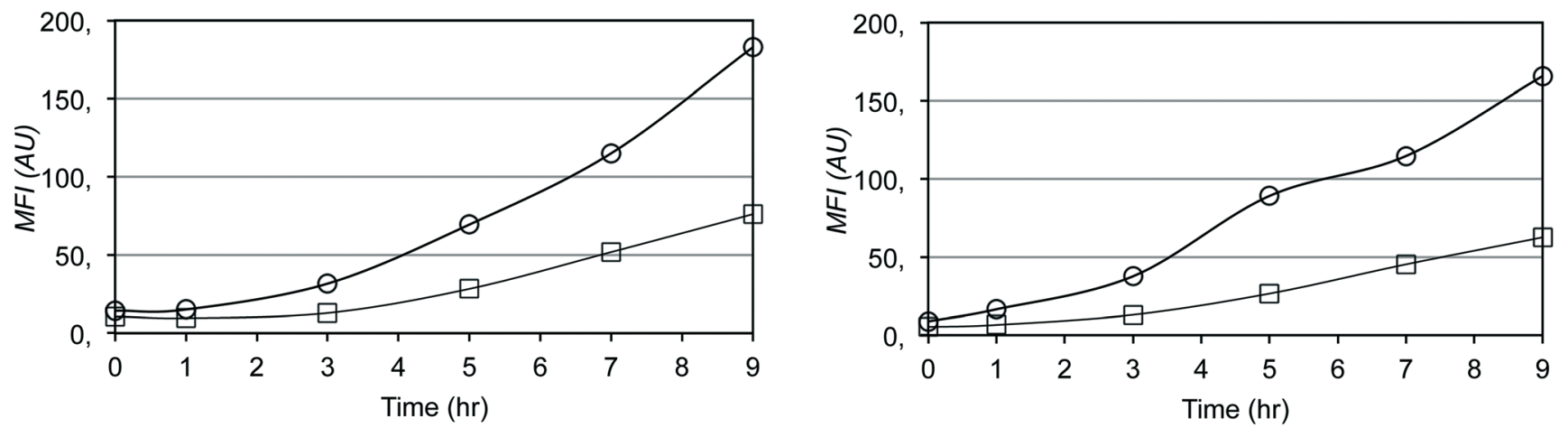

Fig. 4 Time-dependent induction of caspase activities by compound 5 in NT2/D1 and THP-1 cells. Time dependent induction of caspases 3/7 by analogue 5 was observed in accordance with DNA fragmentation seen in Fig. 4. Caspase 8 (- - -) and caspase 9 (- $\square-)$ differential activation was also monitored for both cell lines in the presence of analog 5. Left panels present graphs obtained for the NT2/D1 cell line and right panels for THP-1 cells, respectively. Cells were incubated with analog $5[25 \mu \mathrm{M}]$ for different time points and caspase $3 / 7$ or caspase 8 and 9 activities were monitored as described under Materials and methods. Dead cells were excluded using 7-AAD staining and activated caspases were detected as a timedependent increase of mean fluorescence intensity per cell (tests run in duplicate, 5000 events per sample). 
NT2/D1

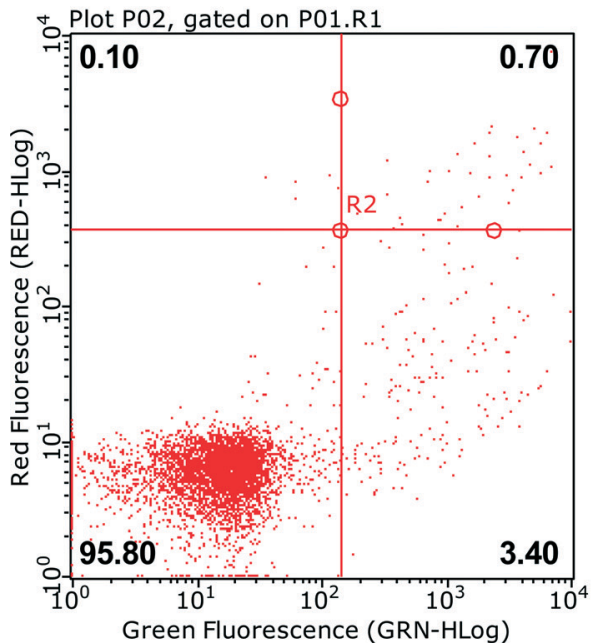

\section{THP-1}

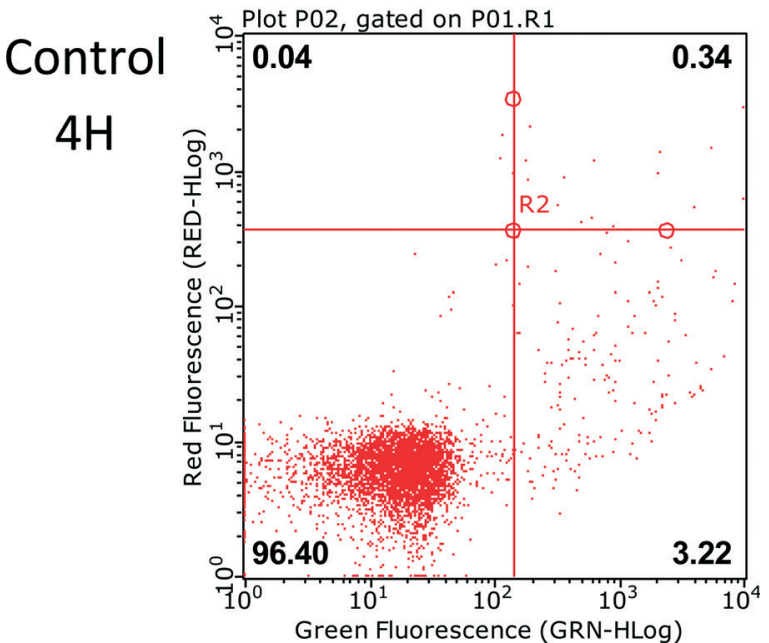

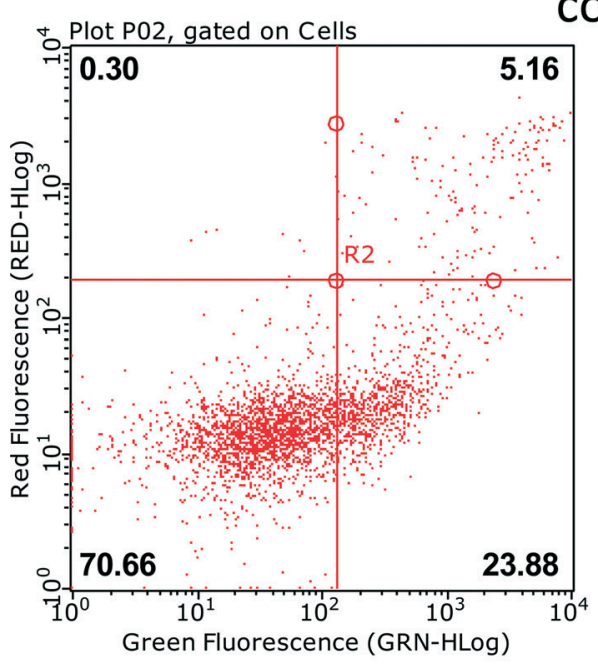

compound \# 5

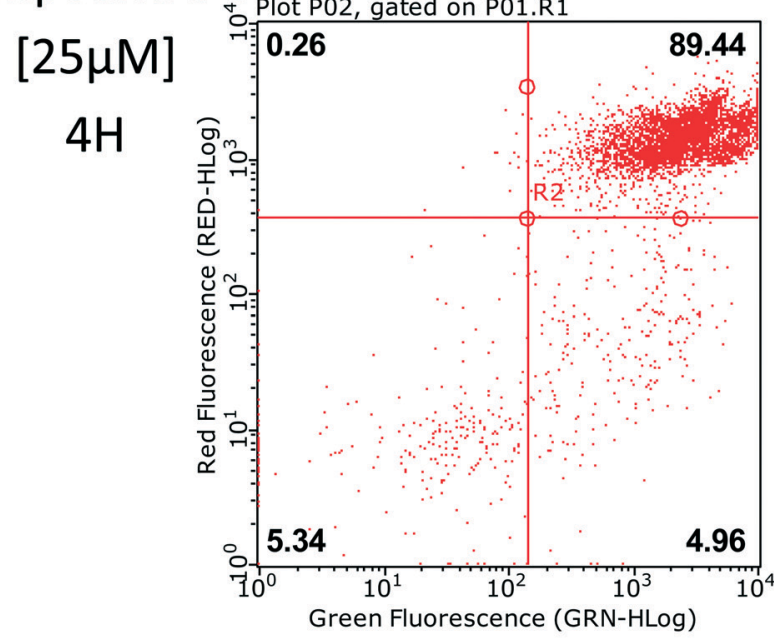

compound \# 5 [25 $\mu \mathrm{M}]+$ Z-VAD-fmk [50 $\mu \mathrm{M}]$
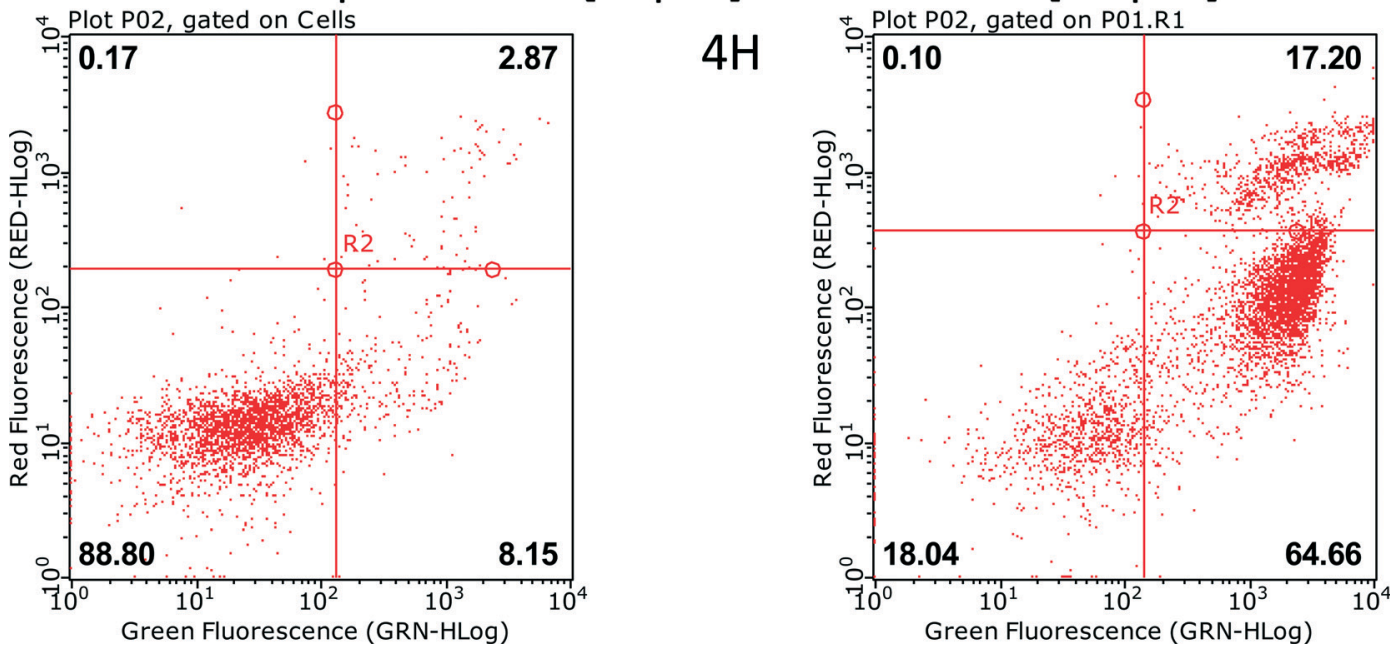

Fig. 5 Different behaviors of the pan caspase inhibitor Z-VAD-fmk in NT2/D1 and THP-1 cells. Representative dot plots obtained after 4 hours of treatment of NT2/D1 and THP-1 cells with or without compound 5 [25 $\mu \mathrm{M}]$ in the presence or absence of the pan caspase inhibitor Z-VAD-fmk [50 $\mu \mathrm{M}$ ]. Cells were labeled with Annexin V/PI as described under M\&M ( $n=3$ independent experiments run in triplicate, 5000 events per sample were analyzed). 
allows the cell to undergo "cellular suicide" in a caspaseindependent fashion in the presence of viral caspase inhibitors, this mechanism could be induced by compound $5 .^{22}$ In addition to being a response to disease, necroptosis has also been characterized as a component of inflammatory diseases. $^{25}$ Thus, the activity of compound 5 on monocytic THP1 cells specifically is plausible, as NT2/D1 cells are not of immune phenotype.

\section{Discussion}

Marine natural products are a valuable source of bioactive compounds, which can be used as leads for drug discovery in various therapeutic areas. ${ }^{23}$ However, like other natural products, marine bioactive compounds are complex in structure and generally don't possess favorable physicochemical properties. Their structure generally needs to be modified in order to obtain synthetically tractable analogues with improved potency, selectivity and improved pharmacokinetic properties. ${ }^{4}$ Following these principles, the results of testing our library of conformationally restricted clathrodin analogues for proapoptotic activity in HepG 2 and THP-1 cancer cells ${ }^{15}$ lead us to test four most potent analogues on additional human cancer (BxPC3) and cancer stem cell (NT2/D1, AsPC-1) models. Comparison of the four analogs for their respective $\mathrm{EC}_{50}$ values on human cells of different phenotypes (Table 1) failed to highlight one of them. Compound 5 bearing no substituent on the indole ring was chosen for a deeper mechanistic study and kinetic experiments were carried out in order to pick the most sensitive cancer phenotype. Among all tested cell phenotypes, the monocytic THP-1 cell line was the only one reacting in less than 5 hours to analog 5 (Fig. 2). After 5 hours of incubation with compound 5 , at a $25 \mu \mathrm{M}$ concentration equivalent to the $\mathrm{EC}_{50}$ for THP-1 cells, $38 \%$ of the cells died by apoptosis. On the other hand on the same time scale, only $6 \%$ of NT2/D1 cells were affected. Such kinetic dissimilarities suggest different targets involved in THP-1 cell death, bearing in mind that all other tested cell phenotypes were responding like NT2/D1 (data not shown). Apoptosis increases the number of hypodiploid sub-G1 cell population, so that an equivalent kinetic profile should have been acquired for 24 hours. The results obtained (Fig. 3) did not confirm a quicker response for THP-1 phenotype; even a slight delay in response was identified. Is such a delay imputable to a difference in the caspase cascade response? But neither for caspase $3 / 7$ nor caspase 8 or caspase 9 could a faster response be observed (Fig. 4). Only the presence of the pancaspase inhibitor Z-VAD-FMK did help to further decipher the mechanism involved (Fig. 5). In 4 hours, the pan caspase inhibitor Z-VAD-fmk was not able to prevent THP-1 annexin-V positive cells; the sum of the lower and upper right quadrants was $82 \%$, a value close to the $95 \%$ observed in the absence of an inhibitor. On the contrary, in NT2/D1 cells, apoptosis was hindered as only $11 \%$ cells presented PS at their surface ( $v s$. $29 \%$ in the absence of an inhibitor). It was demonstrated earlier ${ }^{24}$ that activated caspases inhibit PS flippase during apoptosis, thus blocking the transport of PS from the extra-cellular to the cytoplasmic side imposing PS to stay outside. Furthermore, as recently stated, ${ }^{3}$ scramblase is involved in the first state of activation during apoptosis, then flippase is inactivated to induce all PS to stay outside. Considering the results obtained in the presence of the pan-caspase inhibitor Z-VAD-fmk, one could assume scramblase to be the target of analog 5. But for the NT2/D1 cell line, the cell surface PS induced by compound 5 is highly reduced by the action of the caspase inhibitor, indicating that, for that cellular phenotype, the transport of PS from the extra-cellular to the cytoplasmic side is still effective even in the case of an activated scramblase. For the THP-1 cells, in the presence of compound 5 and the caspase inhibitor Z-VAD-fmk, PS is highly present outside the cells indicating that in the presence of the studied clathrodin analogs, PS extracellular exposure is really dependent on the cellular phenotype. As mentioned earlier, a necroptosis induction could be involved here. Necroptosis is a programmed cell death pathway recently identified by Degterev et al. ${ }^{25}$ Necroptosis is a viral defense mechanism allowing the cell to undergo "cellular suicide" in a caspase-independent fashion in the presence of viral caspase inhibitors. ${ }^{25}$ The clathrodin analogues could also disturb bidirectional trafficking of the membrane between the cell surface and cytoplasm following the model of Lee, S. H. and colleagues, where PS externalization reflects $\mathrm{Ca}^{2+}$-mediated trafficking of many vesicles to the cell surface. ${ }^{2}$ These authors suggest that apoptotic PS externalization results from a two-step process that begins with the internalization of the plasma membrane as cells shrink during apoptosis, forming intracellular vesicles that are then trafficked back to the cell surface in a $\mathrm{Ca}^{2+}$-dependent manner. According to their new model for PS externalization during apoptosis, they identified an altered step that could as well be applied for caspase independent apoptotic PS exposure when compared to the CSC cell line NT2/D1.

The original results described therein with a new set of clathrodin analogues demonstrate that in a monocytic cancer phenotype, PS exposure can be a caspase independent mechanism. Caspase-independent PS exposure was described earlier in primary $\mathrm{T}$ cells during apoptosis induced by stimuli that do not trigger death receptors. ${ }^{26}$ Today different ways of action can be supported. The first is the one suspected by S. Nagata $^{3}$ as the scramblase activity seems highly involved in the monocytic cell lineage. Secondly, again in accordance with monocytic cellular phenotype, intracellular vesicles trafficking back to the cell surface can be involved if one follows the model of Lee, S. H. and colleagues ${ }^{2}$ so that further studies are needed to distinguish between these two models. Thirdly, necroptosis induction in THP-1 cells cannot be excluded. Thus apoptotic PS externalization induced by clathrodin analogue 5 on blood cancer cells could result from a caspase independent mechanism specific to monocytic cell lineage and reflect either scramblase modulation, disturbed bidirectional trafficking of the membrane between the cell surface and cytoplasm or necroptosis induction, highlighting 
analogue 5 as an interesting new pharmacological tool. In summary, the presented results permitted the discovery of a new set of clathrodin analogs with pro-apoptotic activities on different cancer stem cells (NT2/D1, AsPC-1 and BxPC-3) with differential kinetic properties on immune cells (THP-1).

\section{Conflict of interest}

The authors declare that they have no conflict of interest.

\section{Acknowledgements}

This study has been supported by the European Union FP7 Integrated Project MAREX: Exploring Marine Resources for Bioactive Compounds: From Discovery to Sustainable Production and Industrial Applications (Project No. FP7-KBBE-20093-245137), the Slovenian Research Agency (Grant No. P1-0208) and COST action: Chemical Approaches to Targeting Drug Resistance in Cancer Stem Cells (COST-STSM-CM1106-20119).

\section{References}

1 M. E. Ramos-Nino, ISRN Oncol., 2013, 2013, 697521.

2 S. H. Lee, X. W. Meng, K. S. Flatten, D. A. Loegering and S. H. Kaufmann, Cell death Differ., 2013, 20, 64-76.

3 K. Segawa, S. Kurata, Y. Yanagihashi, T. R. Brummelkamp, F. Matsuda and S. Nagata, Science, 2014, 344, 1164-1168.

4 F. E. Koehn and G. T. Carter, Nat. Rev. Drug Discovery, 2005, 4, 206-220.

5 J. J. Morales and A. D. Rodriguez, J. Nat. Prod., 1991, 54, 629-631.

6 S. Forenza, L. Minale, R. Riccio and E. Fattorusso, J. Chem. Soc. D, 1971, 1129-1130.

7 E. E. Garcia, L. E. Benjamin and R. I. Fryer, J. Chem. Soc., Chem. Commun., 1973, 78-79.

8 J. Kobayashi, Y. Ohizumi, H. Nakamura and Y. Hirata, Experientia, 1986, 42, 1176-1177.

9 A. L. Rentas, R. Rosa, A. D. Rodriguez and G. E. De Motta, Toxicon, 1995, 33, 491-497.

10 A. Al-Sabi, J. McArthur, V. Ostroumov and R. J. French, Mar. Drugs, 2006, 4, 157-192.

11 J. J. Richards, T. E. Ballard and C. Melander, Org. Biomol. Chem., 2008, 6, 1356-1363.
12 F. Cafieri, R. Carnuccio, E. Fattorusso, O. TaglialatelaScafati and T. Vallefuoco, Bioorg. Med. Chem. Lett., 1997, 7, 2283-2288.

13 R. Rosa, W. Silva, G. Escalona de Motta, A. D. Rodriguez, J. J. Morales and M. Ortiz, Experientia, 1992, 48, 885-887.

14 A. Žula, D. Kikelj and J. Ilaš, Tetrahedron Lett., 2014, 55, 3999-4001.

15 T. Tomašić, D. Nabergoj, S. Vrbek, N. Zidar, Ž. Jakopin, A. Žula, Ž. Hodnik, M. Jukič, M. Anderluh, J. Ilaš, M. Sollner Dolenc, J. Peluso, G. Ubeaud-Séquier, C. D. Muller, L. Peterlin Mašič and D. Kikelj, Med. Chem. Commun., 2015, 6, 105-110.

16 N. Zidar, S. Montalvao, Z. Hodnik, D. A. Nawrot, A. Zula, J. Ilas, D. Kikelj, P. Tammela and L. P. Masic, Mar. Drugs, 2014, 12, 940-963.

17 N. Zidar, Z. Jakopin, D. J. Madge, F. Chan, J. Tytgat, S. Peigneur, M. S. Dolenc, T. Tomasic, J. Ilas, L. P. Masic and D. Kikelj, Eur. J. Med. Chem., 2014, 74, 23-30.

18 T. Vanden Berghe, A. Linkermann, S. Jouan-Lanhouet, H. Walczak and P. Vandenabeele, Nat. Rev. Mol. Cell Biol., 2014, 15, 135-147.

19 C. Gunther, E. Martini, N. Wittkopf, K. Amann, B. Weigmann, H. Neumann, M. J. Waldner, S. M. Hedrick, S. Tenzer, M. F. Neurath and C. Becker, Nature, 2011, 477, 335-339.

20 L. Galluzzi, I. Vitale, J. M. Abrams, E. S. Alnemri, E. H. Baehrecke, M. V. Blagosklonny, T. M. Dawson, V. L. Dawson, W. S. El-Deiry, S. Fulda, E. Gottlieb, D. R. Green, M. O. Hengartner, O. Kepp, R. A. Knight, S. Kumar, S. A. Lipton, X. Lu, F. Madeo, W. Malorni, P. Mehlen, G. Nunez, M. E. Peter, M. Piacentini, D. C. Rubinsztein, Y. Shi, H. U. Simon, P. Vandenabeele, E. White, J. Yuan, B. Zhivotovsky, G. Melino and G. Kroemer, Cell death Differ., 2012, 19, 107-120.

21 L. Duprez, E. Wirawan, T. Vanden Berghe and P. Vandenabeele, Microbes Infect., 2009, 11, 1050-1062.

22 C. Becker, J. Hagmann, J. Muller, D. Koenig, O. Stegle, K. Borgwardt and D. Weigel, Nature, 2011, 480, 245-249.

23 T. F. Molinski, D. S. Dalisay, S. L. Lievens and J. P. Saludes, Nat. Rev. Drug Discovery, 2009, 8, 69-85.

24 S. Nagata, R. Hanayama and K. Kawane, Cell, 2010, 140, 619-630.

25 A. Degterev, Z. Huang, M. Boyce, Y. Li, P. Jagtap, N. Mizushima, G. D. Cuny, T. J. Mitchison, M. A. Moskowitz and J. Yuan, Nat. Chem. Biol., 2005, 1, 112-119.

26 C. Ferraro-Peyret, L. Quemeneur, M. Flacher, J. P. Revillard and L. Genestier, J. Immunol., 2002, 169, 4805-4810. 